\title{
Redeeming Mr. Sawbone: compassion and care in the cultures of nineteenth-century surgery
}

\author{
Michael Brown(D)
}

\begin{abstract}
Objective: To complicate understandings of the emotions involved in the surgical encounter.

Methods: I draw on an extensive body of historical material to demonstrate the importance of compassion and sympathy to the professional identities and experiences of early nineteenth-century British surgeons and use this information to reflect on what lessons can be learned for contemporary practice.

Results: This research demonstrates that compassion and sympathy for the patient were a vital part of surgery in the decades immediately preceding the introduction of anaesthesia in the 1840 s and that they played a vital role in shaping the professional identity of the surgeon.

Conclusion: This research suggests that we might develop more complex and inclusive ways of thinking about the doctor-patient relationship in surgery and that we can draw on the experiences of the past to ensure that we take compassion seriously as a vital element of the intersubjective clinical encounter.
\end{abstract}

Keywords: Surgery, Emotion, Compassion, Doctor-patient relationship, History, Professional identity

\section{Background}

Thomas Rowlandson's satirical print Amputation (1793) (Fig. 1) presents a compellingly grim picture of surgery and the surgeon at the turn of the nineteenth century. At the centre of the image is a man having his right leg amputated below the knee. The operation is performed without anaesthesia (which would not be routinely utilised until the 1840s), and to stop him from struggling the patient is held down by force. One leg is tied to the chair, while the other is laid upon a stool. The surgeon on the far left has cut through the flesh and muscle with a knife (the arms of his coat are protected from stains by over sleeves) while another surgeon proceeds to saw through the bone as blood cascades into a bowl. A veritable army of surgeons crowd round. On the floor in front of the patient is a bag of fearsome looking instruments and surrounding him are the skulls and skeletons of animals and humans, both adult and child, as well as a human cadaver laid out on a dissection table. Attached to the wall is a list of 'examined and

Correspondence: michael.brown@roehampton.ac.uk

Department of Humanities, University of Roehampton, London, UK approved surgeons' with such ludicrous names as 'Balloon Thickskull,'Launcelot Slashmuscle' and 'Samuel Sawbone' .

Rowlandson's image is possibly even more shocking to us now than it would have been to his contemporaries, for the cultural divide which separates early twenty-first century surgery from that of the late eighteenth and early nineteenth centuries is chasmal. Nowadays, we expect surgery to be undertaken in a sterile, bounded space by practitioners wearing specialised clothing and using highly -specialised equipment. By contrast, here we have surgeons undertaking a major operation in what appears to be a dissecting room, surrounded by human remains, wearing conventional period dress (including powdered periwigs), and utilising implements that are more reminiscent of the carpenter's shop. However, potentially even more alarming than the appearance of the scene is the disposition of its actors. The patient looks out at the viewer, wide-eyed with agony and terror. His exposed, bare head, lacking the customary wig, is suggestive of his vulnerability. So too are his clothes, which appear disordered. The surgeons, on the other hand, are fully clothed and retain their wigs. They act 


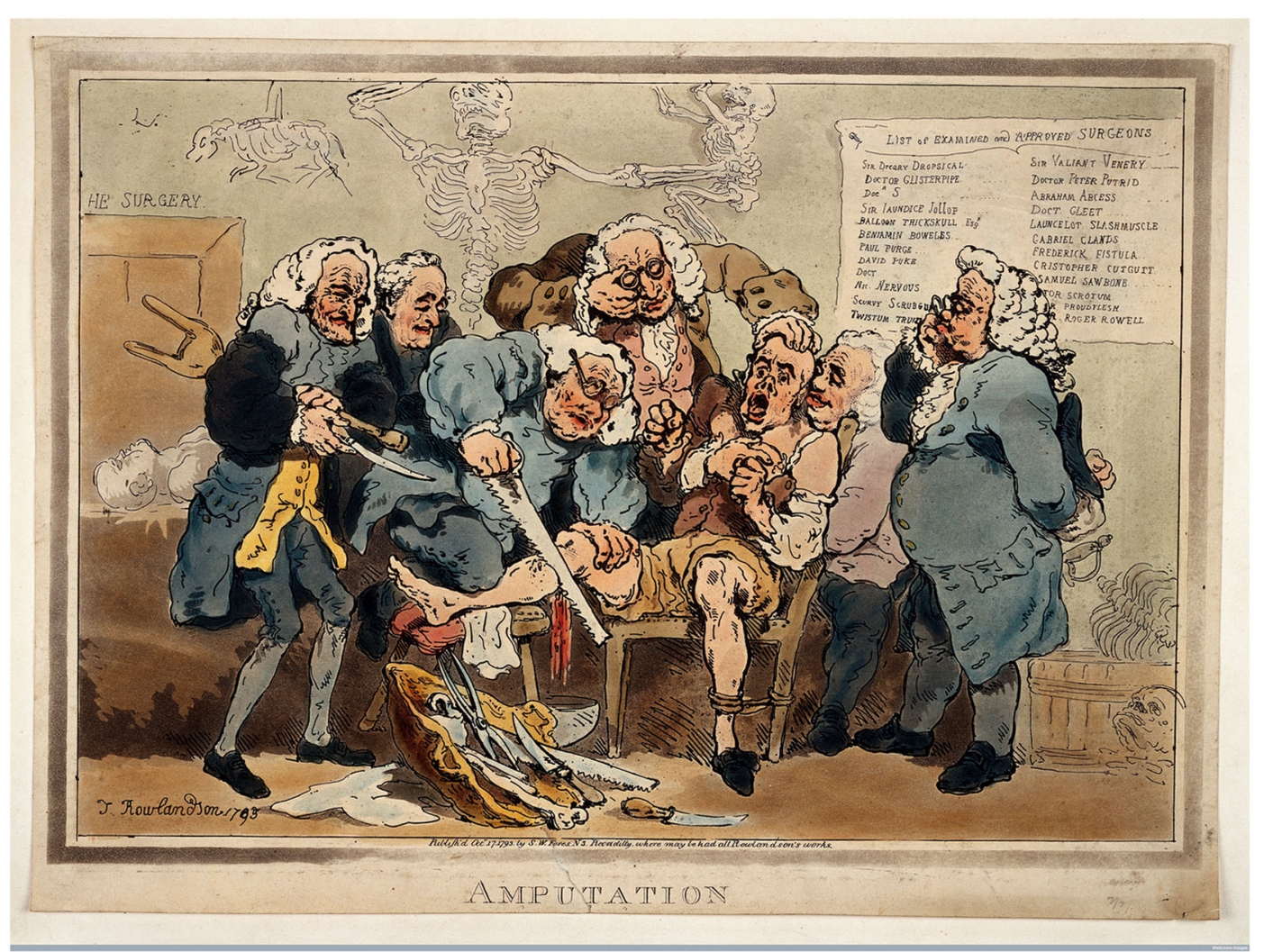

Fig. 1 Thomas Rowlandson, 'Amputation' (1793), Wellcome Library, London

with apparent indifference to the pain they are inflicting. In fact, far from expressing concern for the patient, they are seemingly far more interested in the procedure itself. One looms over the patient to get a closer look, placing his hand upon the poor man's head, while another looks on with a studied nonchalance bordering on disregard. There seems to be a fundamental lack of compassion in evidence here. Cruelty, not care, is the order of the day.

One of the reasons why this image might be so shocking to the modern viewer is that it is part of an unfamiliar visual lexicon. Modern audiences are not used to images depicting medical practitioners as cruel or uncaring. As inhabitants of a post-professional world we tend to accept the idea that doctors and surgeons act for the benefit of society as a whole, not simply in their own pecuniary interest. We are especially not used to seeing negative images of surgeons, who occupy an elevated social and cultural position, perhaps superior even to that of physicians. By contrast, late eighteenth- and early nineteenth-century audiences, who lived in a society where there was no inherent right to free or subsidised healthcare, and in which the authority of the surgeon was far less assured, were schooled in an established tradition of anti-medical satire, which mocked practitioners' self-regard and limited efficacy, as well as their supposed self-interest and disregard for the fate of their patients [1]. They would have recognised with ease the key visual signifiers of Rowlandson's print: the equation of corpulence with greed, of thinness with meanness and grotesqueness of facial feature as a metonym for moral perfidy. They might also have been sensitive to the power dynamics of this image. The surgeons are all old and well-dressed. ${ }^{1}$ The one on the right even wears a dress sword as a marker of (or perhaps pretention to) gentlemanly status. The patient, by contrast, is evidently from a lower social class. If he were a private, fee-paying patient he would almost certainly have been attended in his own residence as opposed to what appears to be 'the surgery'-cumdissection room of a voluntary hospital.

This print might therefore seek to satirise the uncaring attitude of surgeons towards their charitable charges, rather than to their patients in general; we cannot be certain. What is clear, however, is that it tapped into a well-worn stereotype of the surgeon as aloof and insensitive to suffering. As the St George's Hospital surgeon, Benjamin Brodie, told his students in 1820, 'It has been a matter of complaint against our profession, that the being perpetually present at scenes of woe tends to blunt the feelings of our nature, and to render us less capable of sympathizing with the sufferings of others' [2]. We have tended to accept this view. Internet blogs and popular histories exploit the 'blood and 
gore' of pre-modern surgery, while television shows such as BBC's Quacks (2017) play with the image of the early nineteenth-century surgeon as rough, bungling or uncaring. Even serious academic scholarship has tended to emphasise dispassion over compassion. Lynda Payne's work, for example, though immensely valuable, presents dispassion as the primary emotional state of the pre-modern surgeon [3]. In part, a tendency to judge the surgeons of the past comes from surgery's own narrative of constant progress. It has always been a staple of surgical rhetoric to compare the imperfect past with the near perfect present [4]. And in some ways surgeons have internalised this image of themselves, as the dispassionate stoic has morphed into the detached professional.

Then, as now, however, satirical imagery and self-serving histories do not offer us an unmediated window on social reality. Just as modern surgeons do not all conform to the televisual stereotype of the heroic loner, neither were nineteenth-century surgeons necessarily cruel butchers or dispassionate operators. This article demonstrates that the emotional experiences and dispositions of early nineteenthcentury surgeons were complex and nuanced and included scope for a considerable degree of compassion and emotional engagement with patients. What it also suggests is that such lessons from the past can help to encourage a more open and honest discussion of the emotional complexities and challenges of modern-day surgery.

\section{Discussion}

Before we begin, some terminological clarification might be useful. The Oxford English Dictionary defines compassion as 'sympathetic pity and concern for the sufferings or misfortunes of others' [5]. More particularly, compassionate healthcare advocate Dr. Robin Youngson defines it as the 'humane quality of understanding suffering and wanting to do something about it' [6]. The term has a long history and was in use in the early nineteenth century. The Dictionary of the English Language (1828) defined compassion as 'Pity; commiseration' and compassionate as 'Inclined to pity; merciful; exercising compassion' [7]. In a more specific context, the Edinburgh surgeon John Bell wrote in 1801 that, in cases involving a tedious, lingering disease, the surgeon must be every thing to his patient; watchful, friendly, compassionate, cheerful' [8]. In all these cases compassion evokes a feeling, one of pity and sympathy. But in Youngson's definition there is a specific emphasis on action. As he writes, 'It's a motivation, not just a feeling'. The pre-modern usage has a less instrumentalist connotation and was part of a wider constellation of terms relating to the intersubjective experience, the most important and commonly used of which was sympathy.

It is important to note that, in the context of the early nineteenth century, terms such as sympathy and compassion were part of a pervasive culture of sensibility.
Sensibility is a complex concept about which much has been written. However, in brief it can be seen as an intellectual and cultural phenomenon which served to ease social relations through a highly-attuned sensitivity to the feelings of others. In the works of David Hume and Adam Smith, the capacity of the individual to 'feel' through their senses was elaborated into a system of moral philosophy. For our purposes it is notable that one of Smith's early examples in his Theory of Moral Sentiments (1759) concerned the 'chirurgical operation' the sight of which was liable to make people 'faint and grow sick', the 'bodily pain ... occasioned by the tearing of flesh' exciting in them 'a most excessive sensibility' [9]. As this quotation suggests, sympathy could exert a profound physical and physiological influence. Just as contemporaries believed that one part of the body might experience pain or develop disease in sympathy with the disordered actions of another, so too did they believe an individual might be physically overwhelmed, even rendered mortally sick, by an act of imagination.

There were criticisms of this culture of sensibility, with some suggesting that it might be misdirected, exaggerated or even faked [10]. But it remained remarkably resilient and by the early nineteenth century had evolved into what we might term 'Romantic sensibility' with its veneration of honest, heartfelt feeling and its particular emphasis upon the sympathetic treatment of women and children. Indeed, while it is generally thought to have declined from the 1830s onwards, one can see clear traces of its influence in Victorian sentimentality. The leading early nineteenth-century surgeon, Sir Astley Cooper, was said by his nephew and biographer, to be unable to 'supress a tear' when he saw a hungry child in the street and when he read to him the famous scene in the workhouse from Oliver Twist (1837-9) 'he was quite overcome, and, crying like a child, would not suffer me to continue my description of the distressing tale' [11].

It should not be thought, however, that compassion and sympathy in this period were only about feeling and not about agency. To be sure, medical practitioners sometimes talked about feeling things to which their office would not allow them to give full expression. In 1813, for example, the Scottish surgeon Henry Oswald confided to his diary about the distress of dealing with a seriously ill young woman whose despairing father 'groaned in Spirit and writhed with anguish':

These are the scenes which medical men are obliged to behold in apparent coolness whatever may be their inward pain. Perhaps by seeing them so frequently they make less impression on them than others but people are not aware of the anxiety we suffer when a patient is suffering severely and approaching to death, and when every effort of art is in vain. Then we must suppress all feeling, appear composed and endeavour 
to comfort if we do not wish to produce mischief by adding to the alarm which others experience.

Oswald wrote of the 'embarrassing uncertainty of the medical art' in such cases [12]. So too did John Bell, who evoked 'that silent humiliation in the presence of misery, which so well becomes one, who feels that he cannot alleviate the pangs, nor avert the changes, of the scene before him' [13]. Nonetheless, as well as evoking pity, sympathy with suffering was also supposed to encourage benevolence and kindness. Indeed, compassion and sympathy can be said to have been at the heart of the early nineteenth-century surgical encounter.

Returning to Bell's earlier comment about the surgeon being 'every thing' to his patient, the reason he gives for this is that 'the patient lives upon his good looks; it is when his surgeon becomes careless, or seems to forsake him, that he falls into despair' [14]. As this quotation suggests, in the late eighteenth and early nineteenth centuries, it was generally believed that the health of a patient was dependent to a very significant degree on their emotional wellbeing. In an era before complications such as post-operative infection were adequately understood and when the interior operations of the body were largely inaccessible, surgeons often struggled to find reasons why an apparently successful operation or a seemingly mild illness could lead to death. More than this, early nineteenth-century medical orthodoxy perceived little separation between mind and body and, as we have seen, set great store by the operations of sympathy and imagination. As Sir Astley Cooper wrote, 'The mind has great influence over the actions of the body and it often happens after operations that the least discouraging expression will produce fatal effects'. In evidence of this, he cited the case of a Mrs. Shipley who had been operated upon by Cooper's mentor, Henry Cline, for a cancerous breast. 'She said she was sure she should die', Cooper wrote, and 'immediately after the operation she became almost lifeless and in three hours she died'. As if to prove the inevitability of her demise, he observed that she had made arrangements to hand over her role as mistress of the household, stating: 'All her keys were found marked that there might be no confusion occasioned by her death' [15].

In these circumstances it was imperative that the surgeon do his best to regulate the emotional state of his patient and keep them from sinking into 'low spirits'. In principle, this did not extend to dishonesty. A surgeon was not supposed to mislead a patient about their condition by giving them false hope or sparing them news of unfortunate or dangerous developments. Nonetheless, patients occasionally suspected that this was the case. One of Sir Astley Cooper's female patients, who he was treating for a growth in the breast, wrote to him in 1832:
Could you, Sir think of any thing to afford me relief I should for ever feel extremely thankful, for I must acknowledge that I still feel apprehension of a cancer, and when most troubled with pain am fearful you did not tell me exactly what it really was, therefore dear Sir your candid answer will be very very acceptable to me and greatly ease my mind [16].

Evidently such fears were not unfounded, for in 1822, Mr. Rosenware, a surgeon of Wadebridge in Cornwall, wrote, relative to his patient, 'As Miss Best is extremely anxious and agitated on the subject I have endeavoured as much as possible to keep the real nature of the complaint from her until imperious [sic] changes in it should oblige me to be more explicit, and I still think that the most cautious manner of proposing an operation would be necessary; I have as yet only ventured to hint at it' [17].

Neither were surgeons to be obsequious or false in their manner. Typically, for a society deeply troubled by the idea of insincerity, Benjamin Brodie derided what he called those courtier like manners, those continued attempts to suit the inclination and flatter the self-love of others, by means of which mean persons endeavour to make up for their own Ignorance and want of skill, and which disgrace the dignity of our profession' [18]. Rather, the job of the surgeon was to manage his patient through an earnest 'gentleness of manner'. This was important enough in the consultative relationships that composed much of the surgeon's workload, but it was especially vital when it came to the prospect of operative surgery. Surgery is a troubling enough prospect in the modern day but, in the absence of anaesthesia and modern forms of infection control, it was infinitely more so in this period. As Cooper suggested, 'Patients having a natural dislike to operations, feel still more uneasy if they discover any thing in their practitioner's behaviour that makes them apprehend rough treatment' [19]. 'Violence' of manner was 'in all cases bad' and was 'in some attended by fatal consequences'. As a demonstration of this, he referred to the case of a surgeon who, on examining a patient for a compound dislocation of the ankle joint declared 'Carthage must fall. Thereby implying that amputation must be performed'. 'Indeed', Cooper concluded, 'from the rough manner in which he treated his patient there seemed no other chance for the poor fellow's recovery. In this case gentleness might have prevented such an unpleasant circumstance' [19].

Thirty years later, Frederic Skey made a similar point, suggesting that, in the case of a patient about to undergo an operation, it was rare that sympathy does not tell beneficially upon his mind... A peculiar kindness, and in the example of a female or a child, even of tenderness of manner, begets a confidence, which, without betraying weakness or uncertainty, fortifies the patient's mind and reconciles it to the effort' [20]. 
The first thing that was necessary in operative surgery was to judge whether the patient was capable of bearing the procedure. As with Henry Cline's patient and her keys, those patients who were despairing or convinced of their own death should, it was suggested, be spared an operation, whereas the specific fear of the pain produced by the operation itself might be managed. Overall, surgeons of the period argued that one should never operate too rashly. Rather, they should weigh up all the options and, in the words of Cooper, 'make the case your own, and ask if you yourself would submit to it' [21]. When performing the operation, too, gentleness of conduct was a prerequisite. It is often said that early nineteenth-century surgeons were noted for their quickness and, in the case of individuals such as Robert Liston, this was indeed remarked upon. But many surgeons distrusted speed as a marker of skill and saw in it the spectre of self-promotion. Again, as Cooper remarked, 'when performing an operation you are to consider that gentleness is essential to success, and indicates humanity, whereas violence on the contrary is shocking, and tends not a little to the want of success in operations in general. Never regard quickness in operating, tho' weak minds often have that more in view than the safety of the patient' [22].

The patient was, then, to be the sole focus of the surgeon's endeavours and everything possible was to be done to ensure their emotional wellbeing. This even extended to the use and display of surgical instruments. For example, the leading London surgeon John Abernethy cautioned against the use of certain words in theatre. 'Give me the knife Sir', he imagined a surgeon declaring during a trephination; 'good God, what must the patients feeling be, blind folded and hearing give me the knife Sir - Had you not better say give me the Bistoury, a name which not being familiar to the patient would not alarm him' [23]. In another case, the same surgeon entered the operating theatre which had been arranged by a young doctor 'anxious to have everything duly prepared'. On inspection, Abernethy initially declared all to be well but then paused, before throwing a napkin over the instruments and saying it is bad enough for the poor patient to have to undergo an operation without being obliged to see those terrible instrument.' [24].

It should be noted that surgeons did not just seek to accommodate their patients' feelings, they also spoke of their own and the challenges they faced in inflicting pain on their fellow creatures. Occasionally a surgeon might shed a tear during or after a procedure but, for the most part, the rhetoric of surgeons emphasised, in line with Oswald's earlier comments, a degree of emotional restraint. As Everard Home claimed:

An excess of sensibility is of no use \& takes away the power of giving relief. A mother, when the house is on fire will carry her infant through the flames or she may hold her infant to have an operation performed with great firmness \& resolution, \& afterwards when it is over faint away. During an operation, while he is acting for the relief of another, [the surgeon] is putting a restraint on his own feelings. He does not feel the momentous distress he occasions. As there is nothing in Surgery which can soften an unfeeling man so there is nothing to diminish his benevolence or humanity. Every act which he performs is to relieve distress, to remove temporal evils \& to preserve life [25].

\section{Findings}

As the above examples have hopefully demonstrated, the early nineteenth-century surgeon did not necessarily conform to the stereotype of the unfeeling sawbones. Many of these observations are drawn from the writings of some of the leading practitioners of their day. Moreover, many are taken from lectures given to surgical pupils. What this suggests is that compassionate care was not simply the preserve of a few individuals but was part of the broader culture of early nineteenth-century surgery. This is not to say that some practitioners did not behave in a manner which was rough or emotionally insensitive. Home himself acknowledged that 'the harshness of manner \& want of temper of some' surgeons accounted for 'why this reflection had been applied to the profession at large' [26]. What is clear, however, is that in the early decades of the nineteenth century prominent surgeons and surgical lecturers were endeavouring to shape a personal and collective professional identity based not simply upon scientific respectability but also upon humanity, sympathy and compassion. We need to see this development in the context of professional power relations. The period from 1800 to 1850 witnessed a remarkable increase in the social and cultural status of surgery. From relatively low-status practitioners associated with barbers and even butchers, mid-nineteenth-century surgeons developed into remarkably self-confident practitioners on the cusp of displacing physicians from the pinnacle of the medical profession. That this process was already well underway before the advent of anaesthesia suggests that this rise in status had less to do with scientific breakthroughs than with self-presentation and social performance. By promoting the values of sympathy and compassion, surgeons were appealing to a culturally resonant set of values centred on the Romantic ideals of sensibility. In so doing they not only distanced themselves from the stereotype of the butcher, but also from the classic image of physicians as a professional elite concerned with the more acquisitive and fashionable dimensions of social prestige. The surgeon, though he may have come from a more humble background than the physician was, then, no less of a gentlemen. Indeed, in terms of the cultures of sensibility, he may have been more so. 
Given the readership and objectives of this journal it might well be worth concluding this account of compassionate care in the early nineteenth-century by reflecting briefly on its implications for contemporary surgical practice. Recent years have seen a number of published surgical autobiographies, such as Henry Marsh's Do No Harm (2014), which attest to the range of emotions experienced by surgeons, from pride and regret to joy and sorrow. Marsh is hardly representative of the average surgeon. An eminent neurosurgeon with a significant public profile, now retired from practice, he is in an especially secure position from which to reflect on his career with emotional honesty. Nonetheless, such popular accounts stand alongside other, more academic, examples of a new-found interest in the emotional dimensions of surgical practice. In the last five years a number of studies have been published which seek to explore the impact of emotions on surgeons' well-being and decision making [27, 28]. What is notable about these studies is that they tend to focus on such issues as stress and 'burnout'. Certainly, according to some observers, one of the main reasons why physicians and surgeons have tended to assume a detached emotional posture is to avoid being overwhelmed by the everyday personal tragedies to which they are inevitably exposed. Another reason, they suggest, is that detachment is necessary to make considered judgements uncoloured by emotional bias $[29,30]$. These expectations are structured by status and, to an extent, gender. Traditionally male roles may value such affective distance but, as Pam Smith's work has shown, the historically feminised profession of nursing, together with other forms of 'caring' work, is still founded upon the value of emotional labour [31].

In their focus on the impact of emotions upon the practitioner, such studies adopt a fundamentally surgeon-centred approach; for the most part they present emotions as something dangerous, a contaminant of the professional persona and a threat to rational decision-making. What they are not is patient-centred; they do not engage with emotions as vital elements in patient-practitioner communication and intersubjective experience. In this way they serve to sustain the model of clinical detachment and emotional disengagement which, as studies show, continues to dominate professional culture and which focusses on 'fixing' problems, rather than treating patients as whole human entities [32].

\section{Conclusion}

It is not unreasonable to assume that the persistence of emotional detachment as a professional ideal is the result of socialisation and education rather than the inherent nature of surgical practice [33, 34]. Surgeons structure their emotional relationships with patients in ways that are expected of them. And there is probably a role for history in this process. Surgeons come to think of detachment as the way it has 'always been', a timeless quality of the practitioner confronted by difficult decisions and emotionally challenging experiences. But as this research demonstrates, detachment is not the way it has 'always been'. It is not the eternal emotional disposition of the surgical operator. Quite the contrary, in fact. At a time when surgery was perhaps at its most dangerous and challenging, in the decades immediately preceding the introduction of anaesthesia, surgeons shaped professional identities which placed emotions at the heart of the doctor-patient relationship and which took them seriously as a vital element in the regulation of health and well-being. As a historian I am not inclined to take such surgical rhetoric and performance at face value, and it is clear that it played a political role in shaping the identity of an inchoate and aspirational professional body. And yet the very existence of such a professional identity might allow us to rethink the place of emotions in surgery and the space we allow for them in our discussions concerning good healthcare practice.

\section{Endnotes}

${ }^{1}$ Their clothes are actually somewhat old-fashioned for the 1790s, but this print was based on a drawing done ten years earlier.

\section{Acknowledgements}

Not applicable

\section{Funding}

This research is funded by a Wellcome Trust Investigator Award in the Humanities and Social Sciences, "A theatre of emotions: the affective landscape of nineteenth-century British surgery" (108,667/Z/15/Z). Aside from initial approval of the project and providing the funding, the Wellcome Trust has not had any role in the design of the study, in the collection, analysis, and interpretation of the data or in writing the manuscript.

Availability of data and materials

Please contact author for data requests.

Authors' contributions

All work for this manuscript was carried out by the sole author.

Ethics approval and consent to participate

Not applicable

Consent for publication

Not applicable

Competing interests

The author declares that he has no competing interests.

Received: 13 October 2017 Accepted: 6 November 2017

Published online: 29 November 2017

References

1. Haslam, F. From Hogarth to Rowlandson: medicine in art in eighteenthcentury Britain. Liverpool: Liverpool University Press; 1996.

2. Royal College of Surgeons, London [RCSL], MS0470/1/2/5, Benjamin Brodie, 'Introductory lecture of anatomy and physiology' (October 1820), f. 20.

3. Payne L. With words and knives: learning medical dispassion in early modern England. Aldershot: Ashgate; 2007.

4. Lawrence C. Democratic, divine and heroic: the history and historiography of surgery. In: Lawrence C, editor. Medical theory, surgical practice: studies in the history of surgery. New York: Routledge; 1992. p. 1-47. 
5. http://www.oxfordreference.com/view/10.1093/acref/9780199571123.001. 0001/m_en_gb0167770. [Accessed 27/07/17].

6. http://heartsinhealthcare.com/point-view-important-compassion-healthcare/. [Accessed 27/07/17].

7. Johnson S, Walker J. A dictionary of the English language. London: William Pickering; 1828. p. 135

8. Bell J. The Principles of Surgery. vol. 1. Edinburgh: T. Cadell; 1801. p. 15.

9. Smith A. The Theory of Moral Sentiments. $3^{\text {rd }}$ ed. London: A Millar; 1767. p. 43.

10. For example, see Markman Ellis, The Politics of Sensibility: Race, Gender and Commerce in the Sentimental Novel. Cambridge: Cambridge University Press; 1996.

11. Cooper BB. The Life of Sir Astley Cooper, Bart., vol. 2. London: J. W. Parker; 1843. p. 93.

12. National Library of Scotland, MS.9003, Diary of H. R. Oswald Snr (1812-13), ff.71-71V.

13. Bell J. Letters on Professional Character and Manners: On The Education of a Surgeon, and the Duties and Qualifications of a Physician. Edinburgh: J. Moir; 1810. pp. 352-3.

14. Bell. Principles of Surgery. p. 15

15. RCSL, MS0008/2/1/7, Casebook of Sir Astley Cooper. In this period, keys were an important signifier of a woman's domestic authority and her domestic responsibilities

16. RCSL, MS0008/2/1/9, Sir Astley Cooper, Annotated copy of illustrations of the Diseases of the Breast.

17. RCSL, MS0008/2/2/3 pt. 1, Astley Cooper, Letters and cases.

18. RCSL, MS0470/1/2/5, Benjamin Brodie, 'Introductory lecture of anatomy and physiology' (October 1820), f. 22.

19. The Lancet, 1:1 (1823) p 4.

20. Skey FC. Operative Surgery. London: J. Churchill; 1850. p. 4.

21. Wellcome Library, London [WL], MS.1860 'Lectures on Surgery by Mr A. P. Cooper [sic]'.

22. WL, MS. Anon. 1860 'Lectures on Surgery by Mr A. P. Cooper [sic]'.

23. J.F. South, RCSL, MSO232/1/5, 'Lectures on Natural and Morbid Anatomy and Physiology delivered by John Abernethy Esq, FRS, in the Anatomical Theatre at St Bartholomew's Hospital in the years 1819 \& 1820. Volume 4th', f. 162

24. Macilwain G. Memoirs of John Abernethy. London: 1854. p. 197.

25. WL, MS.5604, Notes on Twelve Lectures by Everard Home on the Principal Operations of Surgery, ff. 11-2.

26. WL, MS.5604, Anon. 'Notes on Twelve Lectures by Everard Home on the Principal Operations of Surgery', f. 11.

27. Pinto A, Faiz O, Bicknell C, Vincent C. Surgical complications and their implications for surgeons' well-being. Br J Surg. 2013;100(13):1748-55.

28. Orri M, Revah-Lévy A, Farges $\mathrm{O}$. Surgeons' emotional experience of their everyday practice - a qualitative study. PLoS One. 2015;10:11.

29. For example, see Jodi Halpern, From Detached Concern to Empathy: Humanizing Medical Practice (Oxford: Oxford University Press, 2001).

30. Danielle Ofri, What Doctors Feel: How Emotions Affect the Practice of Medicine (Boston: Beacon Press, 2013).

31. Smith P. The Emotional Labour of Nursing: Its Impact on Interpersonal Relations, Management and Educational Environmen (Basingstoke: Palgrave, 1992); idem, The Emotional Labour of Nursing Revisited: Can Nurses Still Care? Basingstoke: Palgrave; 2011

32. Zambrano SC, Chur-Hansen A, Crawford GB. How do surgeons experience and cope with the death and dying of their patients? A qualitative study in the context of life-limiting illnesses. World J Surg. 2013;37(5):935-4.

33. Prentice R. Bodies in formation: an ethnography of anatomy and surgical education. Durham: Duke University Press; 2013.

34. Foster K, Roberts C. The heroic and the villainous: a qualitative study characterising the role models that shaped senior doctors' professional identity. BMC Med Educ. 2016:16:206.

\section{Submit your next manuscript to BioMed Central and we will help you at every step:}

- We accept pre-submission inquiries

- Our selector tool helps you to find the most relevant journal

- We provide round the clock customer support

- Convenient online submission

- Thorough peer review

- Inclusion in PubMed and all major indexing services

- Maximum visibility for your research

Submit your manuscript at www.biomedcentral.com/submit
Biomed Central 